\title{
Review of: "COVID-19 Social Media Information and Misinformation: a Geospatial Analysis of Inter- regional Tweet Disparities in Ghana"
}

\author{
Elia Biganzoli ${ }^{1}$ \\ 1 University of Milan
}

Potential competing interests: The author(s) declared that no potential competing interests exist.

The paper represents a relevant effort to analyze the Social Media contents related to COVID-19. The information provided by Twitter analysis provides relevant tools for the assessment of disease perception among the involved population according to the perspectives of social and community medicine. The evaluation of Inter-regional Tweet Disparities in Ghana could set the basis for targeted interventions in promoting the spread of useful healthcare information as a powerful mean for disease prevention according to standardized quality requirements. Therefore, healthcare planners as well as stakeholders should consider the analysis of Social Media contents with optimal methods for the extraction of relevant information.

Although the paper provides a useful demonstration, there are still open issues to be addressed.

1. There should be a possible selection bias according to the people actually resorting to the use of social media. This situation leads to phenomena well described in the book by David Hand on Dark Data:

Why what you don't know matters https://darkdata.website/. This issue should be carefully faced in the discussion

2. A dynamic portrait of the evolution in Twitter contents compared to the epidemic spread should be also considered to assess the changes in the social perception of the outbreak. An example for the Italian region immediately following China in the outbreak can be found at CoViD-19, learning from the past: $\mathrm{A}$ wavelet and cross-correlation analysis of the epidemic dynamics looking to emergency calls and Twitter trends in Italian Lombardy region. Rivieccio BA, Micheletti A, Maffeo M, Zignani M, Comunian A, Nicolussi F, Salini S, Manzi G, Auxilia F, Giudici M, Naldi G, Gaito S, Castaldi S, Biganzoli E. PLoS One. 2021 Feb 25;16(2):e0247854. doi: 10.1371/journal.pone.0247854. eCollection 2021.

3. A detailed analysis of the nature of accurate information, misinformation and other information should be also performed to understand their originating motivations and tailoring enforcement actions looking to suitable benchmarks and effectiveness measures in the preventive healthcare setting. 